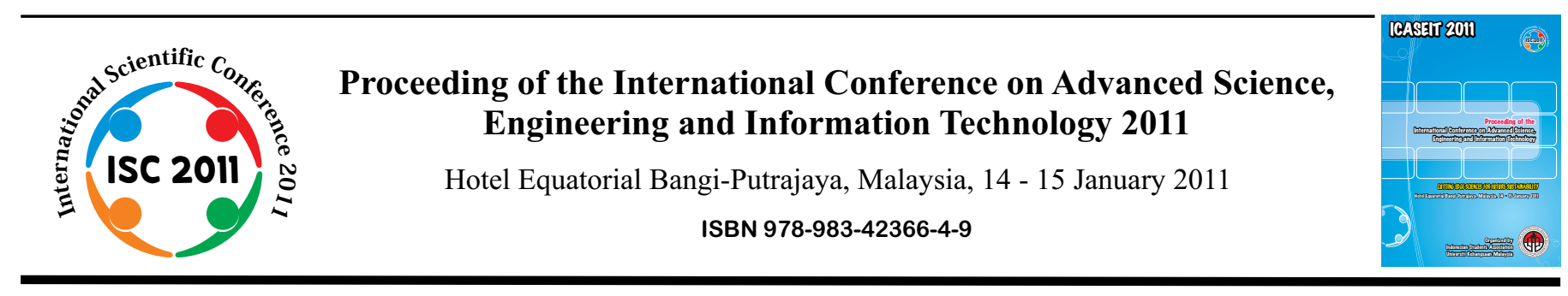

\title{
A Classifier Model based on the Features Quantitative Analysis for Facial Expression Recognition
}

\author{
Amir Jamshidnezhad ${ }^{* \#}$, Md jan Nordin*

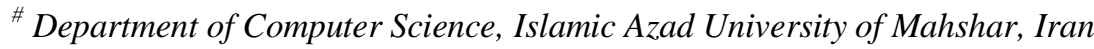 \\ Tel.:+989166126106, E-mail: jamshidnezhad@ftsm.ukm.my \\ *Centre for Artificial Intelligence Technology, Universiti Kebangsaan Malaysia(UKM) \\ Bangi, Selangor Darul Ehsan, 43600, Malaysia \\ Tel.:+60176833173, E-mail: jan@ftsm.ukm.my
}

\begin{abstract}
In recent decades computer technology has considerable developed in use of intelligent systems for classification. The development of HCI systems is highly depended on accurate understanding of emotions. However, facial expressions are difficult to classify by a mathematical models because of natural quality. In this paper, quantitative analysis is used in order to find the most effective features movements between the selected facial feature points. Therefore, the features are extracted not only based on the psychological studies, but also based on the quantitative methods to arise the accuracy of recognitions. Also in this model, fuzzy logic and genetic algorithm are used to classify facial expressions. Genetic algorithm is an exclusive attribute of proposed model which is used for tuning membership functions and increasing the accuracy.
\end{abstract}

Keywords - Facial expression recognition, fuzzy rule based system, genetic algorithm, classifier.

\section{INTRODUCTION}

Over the last decade, the researches on intelligent classification systems have become more active. Intelligent systems in order to classification have been used in a variety of domains such as: Medical, Geology, Biology, Economic, Business, Robotics, Human Computer Interactions (HCI) and Biometrics. Classification of facial expression is one of the important phases of emotions recognition systems. If a computer could recognize the emotions of a user, the user would be able to work and communicate with the computer more easily and efficiently, similar to human-human communications.

There is a variety of applications for facial expression recognition systems: In the animation design, if the animation character simulates the motions based on the human action, the results are more closely to reality. Moreover, in the virtual video communication where the bandwidth does not has the capacity to transfer the video data. In the animation making, the animation character can simulate the human emotions. In the digital cameras for capturing the emotion events and also in the E-commerce for customer relation management (CRM) system, emotion recognition is used to analyze of customer behaviors and motivations.

The well known facial expression model proposed in the research by Ekman and Friesen in 1978, [1]. They showed that there are certain universal emotions from facial expressions which are similar in different cultures. Based on Ekman and Frisen studies, there are at least six basic expressions; Happiness, fear, anger, disgust, surprise, and sadness. Facial Action Coding System

(FACS), a well know collection of facial features for expression recognition, is also introduced by Ekman. Based on FACS, 46 important Action Units (AUs) which are directly related to the movement of eyes, eyebrows and mouth show the facial behaviors [2]. Another important facial feature set is MPEG-4 system which is used for facial expression recognition. MPEG-4 standard specifies 84 feature points on the neutral face which are arranged in the areas like eyes, eyebrows, nose and mouth. Many researchers using the MPEG4 feature points to detect the facial expressions, however, the number of feature points which are associated in the current expression recognition models are different. As the numbers of feature points as 
well as their positions on the face and positions to each other are directly affect the processing time, researchers try to find the optimum number of feature points for using in the expression algorithms. In this paper, the optimum position of the facial feature points is showed which in the expression recognitions are in the high accuracy.

\section{FACIAL EXPRESSION RECOGNITION SYSTEM}

Many Facial Expression Recognition systems have been studied in recent years. Difference in ages, genders and cultures are some reasons which are challenging facial expression recognition. Classifier has important role to analyze the variation such as ages, genders and cultures in the face images for recognizing the six basic emotions from each other [3].

A wide range of classification algorithms (e.g. Hidden Markov Models (HMMs) [4], Bayesian network classifier [5], Support Vector Machine (SVM) [6][7], Neural network (NN) [8][9], PCA [10], LDA [11]) have been applied to the facial expression recognition problem.

In [12], T. Xiang et al. employed Fourier transform to extract features to represent an expression. For further expression processing the Fuzzy C Means (FCM) is used to generate a model for each expression type. A FCM classify the data with the probability between 0 and 1 , so in this classification method, one input object can belong to several class. In this research the Cohen-kanade database was used for training and testing the model. The test samples are matched to the models using the Hausdorff distance to compute dissimilarity values for classification and the best test results was $88.8 \%$. In the proposed method, smile and sadness have the best and worst recognition rates, respectively.

In [13], A. Khanum et al. proposed a hybrid system of fuzzy logic and CBR which has capability to improve the facial expression recognition. In the proposed system, two techniques cover weaknesses of each other's whereas advantages from each other's strengths. They used a simplified feature extraction method using only eight basic facial action elements and the fuzzy rule based system includes 14 rules. They reported the rate of $90.33 \%$ for emotion recognition.

In [14], H. Ebin et al. used fuzzy logic for recognition of four emotions (joy, sadness, anger, and surprise) and reported the rate of $86.7 \%$ for test result. The proposed method in this research was in three main step: 1- preprocessing for normalized the head motions, 2- feature points extraction from face images, 3- making a fuzzy rule based system for classifying the emotions. In this research the recognition of expression is classified in two phases: a) Recognition of changes for different parts of facial features and b) Recognition of facial expression based on the phase (a).

\section{A. Feature Extraction}

The following diagram in Fig.1 indicates briefly the relevant processing stages of proposed facial expression recognition system on the Cohn-Kanade database [15]. The face normalization, facial feature extraction as well as classification are important steps that the accuracy of facial expression recognition are depends on them.

Normalization is operated automatically. In order to reduce the complexity of the feature extraction module, only fifteen facial action points is used with the following steps:

\section{1) Marking the Feature Points:}

Marking the feature points manually based on psychological surveys and literature studies. In this phase fifteen feature points is selected from G. Lipori's study [16] which includes manual annotations of 59 facial fiducial points on the CohnKanade database.

\section{2) Normalizing}

Normalizing includes removing effect of object distances from the camera and the head rigid motions. The distance between corners of two eyes is defined as the principal parameter of measurement. Therefore all feature points are normalized based on this distance. For removing the head rigid motions the amount of horizontal rotation of the face, $\theta$ is determined using the following equation:

$$
\theta=\operatorname{Arctan}\left(\frac{y 1-y 5}{x 1-x 5}\right)
$$

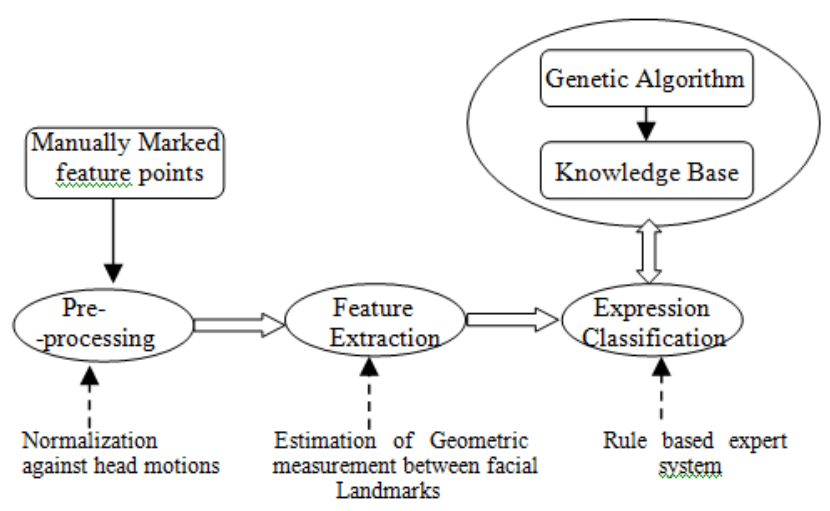

Fig. 2 Facial Expression Recognition Framework

\section{B. Statistical Analysis to Extract the Features}

In order to find the most effective features movements between the selected points, from the first frame to last frame, statistical analysis besides the psychological methods is used. Also the proposed features are experimented in the expression recognition model; therefore, more accurate results than the current researches are obtained. As the results is shown in the following figures, fifteen feature points, include thirteen distances between pairs of points, have most changes from the first image frame (neutral) to last image frame (emotional) in the six facial expressions. Table. 1 shows the position of the selected points on the facial expressions. Fig 2, 3 and fig. 4 show the most effective distances between the feature points which change between neutral to emotional expressions on the face. Based on the defined features, proposed classifier technique classifies the facial expressions.

\section{Genetic-Fuzzy Classifier}

With the purpose of making better performance of fuzzy rule based system, Genetic learning Processes designed for parameter optimization [17]. In this paper, fuzzy rule based system combined with the Genetic Algorithm is proposed to reduce the time needed for training and to improve the robustness of the system under adverse conditions. 
TABLE. I

Selected distances between pairs of the points based on the vertica position (Y) and horizontal position (X)

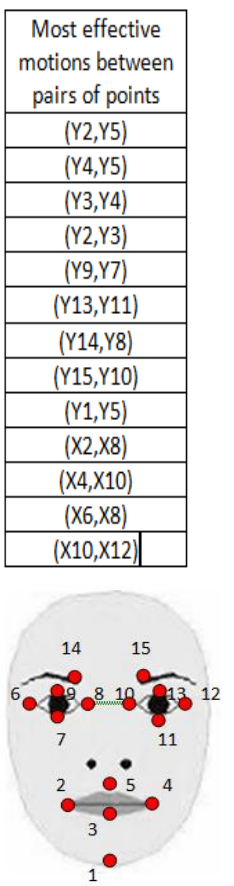

Fig. 2 Selected 15 facial feature points
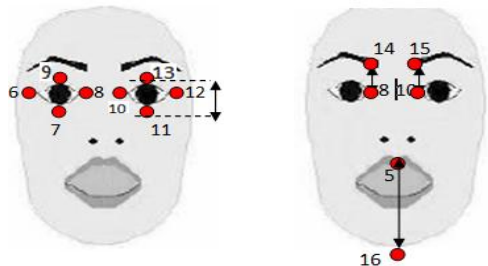

Fig. 3 Extracted features on the eyes, eyebrows and chin

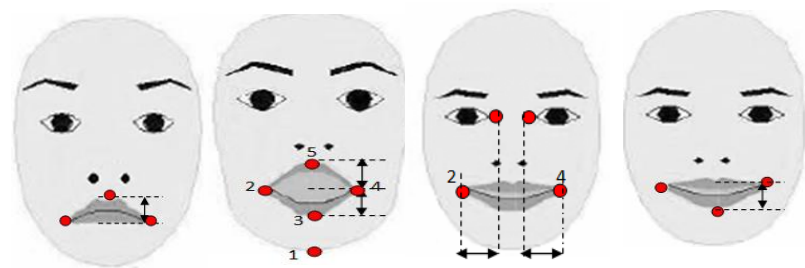

Fig. 4 Extracted features on the mouth and lip

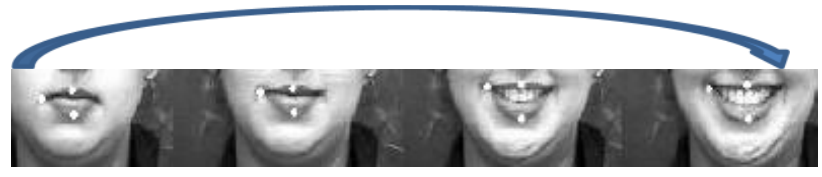

Fig. 5 Extracted feature on the mouth between neutral frame to emotional frame.

This integration is especially useful in classification problems where it is hard to find crisp distinction between two classes.

In this paper, the core of Expression Recognition Module is a Mamdani-type fuzzy rule based system. The knowledge base $(\mathrm{KB})$ is divided into two components: Fuzzy rule base and Genetic Algorithm.

- Fuzzy Rule Based System

Rule base is made based on the empirical studies of changing the feature extracted from neutral to one emotion expression. Inference system, based on the rules classifies input feature vectors into one of the six basic emotions.
For each feature extracted, five linguistic variables will be defined: a) Very small, b) Small, c) Medium, d) Large, e) Very large. Example of rule is showed as follow:

If f1: medium And f2: small And f3: medium And f4: medium And f5: very large And f6: large then expression is Happiness.

In this paper, Gaussian membership function is used to map inputs to membership values. Gaussian membership function is one of the fuzzy membership functions that is often used to represent vague; linguistic terms is the Gaussian which is given by [18]:

$$
\mu(x)=\exp \left(-\left(\frac{x-b}{a}\right)^{\beta}\right.
$$

Where $b$ and $a$ are the centre and width of the membership functions, respectively. Factor $\beta$ is used to change the Gaussian function shape. For $\beta=2$ is obtained the classical shape of Gaussian function and the closely triangular or trapezoidal shapes are obtained for suitable factors $\beta=0.5$ and $\beta=5$.

\section{- Genetic Algorithm}

In the proposed system, a Genetic Algorithm (GA) based method for the modification of membership functions of antecedent fuzzy sets in fuzzy rules for classification problem is developed. The modification of the shapes of membership functions improved the performance of the classification system. The process of GA is as follow:

1)Define the Choromosome length: There are seven extracted features (f1- f6) and each of them have five linguistic variables (VS, S, M, L and VL) and also each Gaussian membership function has three parameters, so we have $90\left(6^{*} 5^{*} 3\right)$ Genes in each chromosome $\mathrm{Ci}=(\mathrm{G} 1, \mathrm{G} 2, \ldots$ , Gn) .

2)Fitness Function: Fitness functions is derived from the following objective function:

$$
\operatorname{Min} f(x)=1-\sum_{k=1}^{c}\left(\frac{m k}{n k}\right)
$$

Where the $\boldsymbol{m} \boldsymbol{k}$ is the numbers of correct classification for selected training data include six classes and, $n k$ is the total of training data.

3)Parent choromosome: The Roulette wheel technique is used for selecting the pairs of chromosomes from the current population.

4)Crossover: Two points crossover technique is used for creating a pair of offspring. The position of the points of crossover is selected randomly but separately for the genes which are related to the centre and width and beta parameters. It means the crossover operates just on the centres genes without changing in the width and beta parameters and again operates just on the width and finally operates on the beta parameters.

Cross over probability $(\mathrm{Pc})$ is changeable based on the rate of fitness improvements .

5)Mutation: Mutation apply with the probability of $(\mathrm{Pm})$ at the beginning, For the purpose of the mutation a randomly decimal number $(+\varepsilon$ or $-\varepsilon)$ will be added to the gene.

(After mutation: limited in the allowed range) $\mathrm{Gi}=\varepsilon+\mathrm{Gi}$ (Before mutation) 
6)Reproduction: Best chromosome which have the best fitness, is selected between the offsprings and the primary population. The new population is created based on the best chromosomes.

7)Termination conditions: The process of step of " 3 " to " 6 " will be repeated until the termination criterion will be satisfied. Terminating conditions are: 1) Fixed number of generations reached, 2) The highest ranking solution's fitness is reached, 3) Successive iterations no longer produce better fitness values.

\section{CONCLUSIONS}

Facial expression recognition has the important role in the human-computer interaction (HCI) systems. The research on emotion recognition through facial images indicates the requirement of two desired directions:

1- On the identifying the most effective facial features and

2- On interpreting the face emotion through the processed facial features.

Although there is a wide range of methods commonly used to facial expression recognition, however the variety of facial expression and less attention to the feature extraction are caused the complexity in the recognition systems. Therefore, objective in this research is development of facial expression recognition systems using Genetic Algorithm and Fuzzy rule based System (GAFs) in order to reduce the complexity and also performance improvement.

\section{REFERENCES}

[1] P. Ekman, W.V. Friesen, The facial action coding system. Palo Alto, Calif. Consulting Psychologists Press, 1978.

[2] Y. Li Tian, T. Kanade and J. F. Cohn, Recognizing Action Units for Facial Expression Analysis, Robotics Institute, Carnegie Mellon University, Pittsburgh, PA 15213, 1999.

[3] B. Fasela, J. Luettin, Automatic facial expression analysis: a survey, Pattern Recognition, vol. 36, 2003, pp. 259-275.

[4] M. Pardàs, A. Bonafonte, and J. Landabaso, Emotion recognition based on MPEG4 facial animation parameters, in: Proceedings of IEEE ICASSP, 2002

[5] I. Cohen, N. Sebe, F. Cozman, M. Cirelo, and T. Huang, Learning Bayesian network classifiers for facial expression recognition using both labelled and unlabeled data, in: Proc. of the 2003 IEEE CVPR, 2003.

[6] J.M. Susskind, G. Littlewort, M.S. Bartlett, J. Movellan, and A.K. Anderson, Human and computer recognition of facial expressions of emotion, Neuropsychologia, vol. 45, 2007, pp. 152-162

[7] A. Geetha, V. Ramalingam, and S. Palanivel, Facial expression recognition, A real time approach, Expert Systems with Applications,vol. 36, 2009, pp. 303-308

[8] L. Ma, K. Khorasani, Facial expression recognition using constructive feed forward neural networks, IEEE Trans. Syst. Man Cybern,2004

[9] H. Seyedarabi, A. Aghagolzadeh, and S. Khanmohammadi, Recognition of Six Basic Facial Expressions by Feature-Points Tracking using

[10] S.Dubuission, F. Davoine, and M. Masson, A Solution for facial expression representation and recognition, Signal Proccessing: Image Communication, vol.17, 2002, pp.657-673.

[11] X. Chen, T. Huang, Facial expression recognition: A clustering-based approach, Pattern Recognition Letters, vol. 24, 2003, pp.1295-1302.
[12] T. Xiang, M.K.H. Leung, and S.Y. Cho, Expression recognition using fuzzy spatio-temporal modeling, Pattern Recognition, vol. 41,2008, pp. 204-216

[13] A. Khanum, M. Muftib, M.Y. Javed, and M.Z.Shafiq, Fuzzy casebased reasoning for facial expression recognition, Fuzzy sets and systems, 2009, pp. 231-250

[14] H. Ebine, Y. Shiga, M. Ikeda and O. Nakamura," Automatic Detection of Reference Face and The Recognition of Transition of Facial Expressions, Transactions of The Institute of Electrical Engineers of Japan vol. 121, no. 10, 2001.

[15] T. Kanade, J. Cohn, and Y. Tian. Comprehensive database for facial expression analysis, 2000.

[16] G. Lipori, [manual annotations of facial fiducial points], LAIV laboratory, University of Milan, web url: http://lipori.dsi.unimi.it/download.html.

[17] F. Herrera, Genetic Fuzzy Systems: Status, Critical Considerations and Future Directions, International Journal of Computational Intelligence Research, vol.1, No.1, 2005.

[18] A. Pieczynski, Application of General Gaussian Membership Function for the Fuzzy Model, Artificial intelligence and soft computing - (ICAISC 2004), 2004, pp. 350-355. 